Open J. Math. Anal., Vol. 2(2018), No. 2, pp. 156 - 171

Website: https://pisrt.org/psr-press/journals/oma/

ISSN: 2616-8111 (Online) 2616-8103 (Print)

http://dx.doi.org/10.30538/psrp-oma2018.0025

\title{
GLOBAL SOLUTION AND ASYMPTOTIC BEHAVIOUR FOR A WAVE EQUATION TYPE $p$-LAPLACIAN WITH MEMORY
}

\author{
CARLOS ALBERTO RAPOSO ${ }^{1}$, ADRIANO PEDREIRA CATTAI, \\ JOILSON OLIVEIRA RIBEIRO
}

\begin{abstract}
In this work we study the global solution, uniqueness and asymptotic behaviour of the nonlinear equation

$$
u_{t t}-\Delta_{p} u=\Delta u-g * \Delta u
$$

where $\Delta_{p} u$ is the nonlinear p-Laplacian operator, $p \geq 2$ and $g * \Delta u$ is a memory damping. The global solution is constructed by means of the Faedo-Galerkin approximations taking into account that the initial data is in appropriated set of stability created from the Nehari manifold and the asymptotic behavior is obtained by using a result of P. Martinez based on new inequality that generalizes the results of Haraux and Nakao.
\end{abstract}

Mathematics Subject Classification: 35B40, 35L70, 35A01, 74DXX.

Key words and phrases: p-Laplacian operator; global solution; asymptotic behaviour; memory.

\section{Introduction}

Throughout this paper we omit the space variable $x$ of $u(x, t)$, simply denote $u(x, t)$ by $u(t)$ when no confusion arises and $c$ denotes various positive constants depending on the known constants and may be different at each appearance. We use the Sobolev space with its properties as in R. A. Adams [1] and H. Brezis [2]. Let $\Omega \in \mathbb{R}$ be a open and bounded interval, $2 \leq p<\infty$ and $p^{\prime}$

Received 03-11-218. Accepted 05-12-2018.

1 Corresponding Author

(C) 2018 Carlos Alberto Raposo, Adriano Pedreira Cattai and Joilson Oliveira Ribeiro. This is an open access article distributed under the Creative Commons Attribution License, which permits unrestricted use, distribution, and reproduction in any medium, provided the original work is properly cited. 
such that $\frac{1}{p}+\frac{1}{p^{\prime}}=1$. The duality pairing between the space $W_{0}^{1, p}(\Omega)$ and its dual $W^{-1, p^{\prime}}(\Omega)$ will be denoted using the form $\langle\cdot, \cdot\rangle_{p}$. According to Poincarè's inequality, the standard norm $\|\cdot\|_{W_{0}^{1, p}(\Omega)}$ is equivalent to the norm $\|\nabla \cdot\|_{p}$ on $W_{0}^{1, p}(\Omega)$. Henceforth, we put $\|\cdot\|_{W_{0}^{1, p}(\Omega)}=\|\nabla \cdot\|_{p}$. We denote $\|\cdot\|_{L^{2}(\Omega)}=|\cdot|_{2}$ and the usual inner product by $(\cdot, \cdot)$. We denote the $p$-Laplacian operator by $\Delta_{p} u$, which can be extended to a monotone, bounded, hemicontinuous and coercive operator between the spaces $W_{0}^{1, p}(\Omega)$ and its dual by

$$
\begin{aligned}
& -\Delta_{p}: W_{0}^{1, p}(\Omega) \rightarrow W^{-1, p^{\prime}}(\Omega) \\
& \left\langle-\Delta_{p} u, v\right\rangle_{p}=\int_{\Omega}|\nabla u|^{p-2} \nabla u \nabla v \mathrm{~d} x
\end{aligned}
$$

Nonlinear hyperbolic problems involving the $p$-Laplacian are becoming the object of increasing interest only in recent years. The existence of a global solution for wave equation of $p$-Laplacian type

$$
u_{t t}-\Delta_{p} u=0
$$

without an additional dissipation term is an open problem. For $n=1, \mathrm{M}$. Derher [3] proved the local in time existence of solution and showed by a generic counter-example that the global in time solution can not be expected.

Adding a strong damping $\left(-\Delta u_{t}\right)$ in (1) the well-posedness and asymptotic behavior was studied by J. M. Greenberg [4. In fact, the strong damping plays an important role on the existence and stability for $p$-Laplacian wave equation see for instance for $n \geq 2$ [5, 6, 7, 8, 9, 10, 11, 12. Nevertheless, if the strong damping is replaced by a weaker damping $\left(u_{t}\right)$, then global existence and uniqueness are only know for $n=1,2$, see [13, 14]. For the intermediary damping given by $\left((-\Delta)^{\alpha} u_{t}\right)$, with $0<\alpha \leq 1$ in [15] was proved the global solution depending on the growth of a forcing term. The background of these problems are in physics, especially in solid mechanics.

From what we know this is the first time that a alternative damping for wave equation with the $p$-Laplacian operator is considered. In this work we consider a memory damping, acting only on $\Delta u$ given by the usual convolution

$$
g * \Delta u(x, t)=\int_{\Omega} g(t-s) \Delta u(x, s) \mathrm{d} s
$$

with the kernel $g$ as real-valued function.

We have interest in proving the existence of a global solution and energy decay to the problem

$$
\begin{gathered}
u_{t t}-\Delta_{p} u=\Delta u-g * \Delta u \quad \text { in } \quad \Omega \times[0, \infty), \\
u(x, 0)=u_{0}(x), u_{t}(x, 0)=u_{1}(x), x \in \Omega, \\
u(x, t)=0 \quad \text { on } \partial \Omega \times[0, \infty) .
\end{gathered}
$$

This paper is organized as follows. Section 2 deals with the potential well, we introduce the stability set for the problem. In the Section 3 we introduce 
some notations and preliminaries results. In the section we introduce a suitable Galerkin basis. In the Section 5 we prove the existence of solution by FaedoGalerkin method and finally in the Section 6 we use the result of P. Martinez [16] that generalizes the results of Haraux [17] and Nakao [18] to prove the energy decay in a appropriate set of stability.

\section{The Potential Well}

It is well known that the energy of a PDE system is, in some sense, split into kinetic and potential energy. Following the idea of Y. Ye [9] we are able to construct a set of stability as follows. We will prove that there is a valley or a well of depth $d$ created in the potential energy. If this height $d$ is strictly positive, we find that, for solutions with initial data in the good part of the well, the potential energy of the solution can never escape the well. In general, it is possible for the energy from the source term to cause the blow-up in finite time. However in the good part of the well it remains bounded. As a result, the total energy of the solution remains finite on any time interval $[0, T)$, which provides the global existence of the solution. We started by introducing the functional $J: W_{0}^{1, p}(\Omega) \rightarrow \mathbb{R}$ by

$$
J(u)=\frac{1}{p}\|\nabla u\|_{p}^{p}-\frac{1}{2} \int_{\Omega}\left(g *|\nabla u|^{2}\right)(t) \mathrm{d} x .
$$

For $u \in W_{0}^{1, p}(\Omega)$ we define the functional

$$
J(\lambda u)=\frac{\lambda^{p}}{p}\|\nabla u\|_{p}^{p}-\frac{\lambda}{2} \int_{\Omega}\left(g *|\nabla u|^{2}\right)(t) \mathrm{d} x, \quad 0<\lambda \leq 1 .
$$

Associated with the $J$ we have the well known Nehari Manifold given by

From (6) we get

$$
\mathcal{N} \stackrel{\text { def }}{=}\left\{u \in W_{0}^{1, p}(\Omega) /\{0\}:\left[\frac{\mathrm{d}}{\mathrm{d} \lambda} J(\lambda u)\right]_{\lambda=1}=0\right\} .
$$

$$
\frac{\mathrm{d}}{\mathrm{d} \lambda} J(\lambda u)=\lambda^{p-1}\|\nabla u\|_{p}^{p}-\frac{1}{2} \int_{\Omega}\left(g *|\nabla u|^{2}\right)(t) \mathrm{d} x,
$$

then

$$
\mathcal{N} \stackrel{\text { def }}{=}\left\{u \in W_{0}^{1, p}(\Omega) /\{0\}:\|\nabla u\|_{p}^{p}=\frac{1}{2} \int_{\Omega}\left(g *|\nabla u|^{2}\right)(t) \mathrm{d} x\right\} .
$$

We define as in the Mountain Pass theorem due to Ambrosetti and Rabinowitz [19,

$$
d \stackrel{\text { def }}{=} \inf _{u \in W_{0}^{1, p}(\Omega) /\{0\}} \sup _{0 \leq \lambda} J(\lambda u) .
$$

It is well-known that the depth of the well $d$ is a strictly positive constant, see [[20], Theorem 4.2], and

$$
d=\inf _{u \in \mathcal{N}} J(u)
$$


In fact, in our problem, the solution of $\frac{\mathrm{d}}{\mathrm{d} \lambda} J(\lambda u)=0$ is

$$
\lambda_{*}=\left[\frac{\frac{1}{2} \int_{\Omega}\left(g *|\nabla u|^{2}\right)(t) \mathrm{d} x}{\|\nabla u\|_{p}^{p}}\right]^{\frac{1}{p-1}} .
$$

We have

$$
\frac{\mathrm{d}^{2}}{\mathrm{~d} \lambda^{2}} J(\lambda u)=(p-1) \lambda^{p-2}\|\nabla u\|_{p}^{p}>0
$$

and then $\lambda_{*}$ is a global minimum.

For $p \geq 2, J\left(\lambda_{*} u\right)<0$, so we introduce the sets

$$
\mathcal{W}_{1}=\left\{u \in W_{0}^{1, p}(\Omega) ; J\left(\lambda_{*} u\right) \leq J(\lambda u) \leq 0\right\}
$$

and

$$
\mathcal{W}_{2}=\left\{u \in W_{0}^{1, p}(\Omega) ; 0<J(\lambda u)\right\}
$$

The potential well is defined by $\mathcal{W}=\left\{u \in W_{0}^{1, p}: J(u)<d\right\} \cup\{0\}$ and partition it into two sets

$$
\begin{gathered}
V=\left\{u \in \mathcal{W}: \frac{1}{2} \int_{\Omega}\left(g *|\nabla u|^{2}\right)(t) \mathrm{d} x \leq\|\nabla u\|_{p}^{p}\right\} \cup\{0\}, \\
W=\left\{u \in \mathcal{W}:\|\nabla u\|_{p}^{p}<\frac{1}{2} \int_{\Omega}\left(g *|\nabla u|^{2}\right)(t) \mathrm{d} x\right\} .
\end{gathered}
$$

We will refer to $V$ as the "good" part of the well and $W$ as the "bad" part of the well. Then we define by $V$ the set of stability for the problem (2)-(4).

\section{Preliminaries}

We introduce the symbols " $\square$ " and " $\diamond$ " which denote the following convolutions respectively

$$
\begin{aligned}
& (g \square h)(t) \stackrel{\text { def }}{=} \int_{0}^{t} g(t-s)|h(t)-h(s)|^{2} \mathrm{~d} s, \\
& (g \diamond h)(t) \stackrel{\text { def }}{=} \int_{0}^{t} g(t-s)(h(t)-h(s)) \mathrm{d} s .
\end{aligned}
$$

We state two basic results, see [21, that will be used in the sequel.

Lemma 3.1. For any functions $g, h \in C([0, \infty], \mathbb{R})$ we have that

$$
\begin{aligned}
(g * h)(t) & =\left(\int_{0}^{t} g(s) \mathrm{d} s\right) h(t)-(g \diamond h)(t) \\
|(g \diamond h)(t)|^{2} & \leq\left(\int_{0}^{t}|g(s)| \mathrm{d} s\right)(|g| \square h)(t)
\end{aligned}
$$


Lemma 3.2. For $g, h \in C([0, \infty], \mathbb{R})$ we have

$$
2(g * h)(t) h^{\prime}(t)=\left(g^{\prime} \square h\right)(t)-g(t)|h(t)|^{2}+\frac{\mathrm{d}}{\mathrm{d} t}\left[\left(\int_{0}^{t} g(s) \mathrm{d} s\right)|h(t)|^{2}-(g \square h)(t)\right]
$$

From now and on, the function $g$ is of exponential type, this is, $g>0$ and $\exists c_{i}>0,(i=0,1)$ such that

$$
-c_{0} g(t) \leq g^{\prime}(t) \leq-c_{1} g(t) \text { and } 1-\int_{0}^{\infty} g(t) \mathrm{d} t<\infty .
$$

The energy of the problem (2)-(4) is defined as

$$
E(t) \stackrel{\text { def }}{=} \frac{1}{2}\left|u^{\prime}(t)\right|_{2}^{2}+\frac{1}{p}\|\nabla u(t)\|_{p}^{p}+\frac{1}{2}\left(1-\int_{0}^{t} g(s) \mathrm{d} s\right)|\nabla u(t)|_{2}^{2}+\frac{1}{2}(g \square \nabla u)(t) .
$$

Now we present the result of P. Martinez [16] on decay rate estimates for dissipative system that will used in the section 6 .

Lemma 3.3. Let $E: \mathbb{R}_{+} \rightarrow \mathbb{R}_{+}$be a non increasing function and $\phi: \mathbb{R}_{+} \rightarrow \mathbb{R}_{+}$ an increasing function such that

$$
\phi(0)=0 \text { and } \phi(t) \rightarrow+\infty \text { as } t \rightarrow+\infty .
$$

Assume that there exist $q \geq 0$ and $A>0$ such that

$$
\int_{S}^{+\infty} E(t)^{q+1} \phi^{\prime}(t) \mathrm{d} t \leq A E(S), 0 \leq S<+\infty .
$$

Then we have

$$
E(t) \leq c E(0)(1+\phi(t))^{\frac{-1}{q}}, \forall t \geq 0 \text { if } q>0
$$

and

$$
E(t) \leq c E(0) e^{-w \phi(t)}, \forall t>0 \text { if } q=0,
$$

where $c$ and $w$ are positive constants independent of the initial energy $E(0)$.

The energy of the problem (2)-(4) is defined as

$$
E(t) \stackrel{\text { def }}{=} \frac{1}{2}\left|u^{\prime}(t)\right|_{2}^{2}+\frac{1}{p}\|\nabla u(t)\|_{p}^{p}+\frac{1}{2}\left(1-\int_{0}^{t} g(s) \mathrm{d} s\right)|\nabla u(t)|_{2}^{2}+\frac{1}{2}(g \square \nabla u)(t) .
$$

\section{The Galerkin basis}

Denote by

$\mathcal{K}_{j}=\left\{K \subset\left\{u \in L^{p}(\Omega):\|u\|_{p}=1\right\}: K\right.$ is compact, symmetric and $\left.\gamma(K) \geq j\right\}$, where $\gamma(G)=\inf \{m: \exists \phi: G \rightarrow \mathbb{R} /\{0\}, \phi$ odd continuous function $\}$ denotes the Krasnoselski genus. In [22] it is proved that

$$
\lambda_{j}=\inf _{G \in \mathcal{K}_{j}} \sup _{u \in G}\|\nabla u\|_{p}^{p}
$$

is a sequence of eigenvalue of the $p$-Laplacian. $-\Delta_{p}: W_{0}^{1, p}(\Omega) \rightarrow W^{-1, p^{\prime}}(\Omega)$ is a monotone, coercive and hemicontinuous operator on $W_{0}^{1, p}(\Omega)$. Minty-Browder 
theorem, see [23], guarantees the existence of a basis $\left(w_{j}\right)_{j=1}^{\infty}$ for $W_{0}^{1, p}(\Omega)$ given by the solution of the stationary problem

$$
\begin{aligned}
-\Delta_{p} w_{j} & =\lambda_{j} w_{j}, \\
w_{j}(0) & =w_{0 j} .
\end{aligned}
$$

Using

$$
W_{0}^{1, p}(\Omega) \subset L^{2}(\Omega) \subset W^{-1, p^{\prime}}(\Omega)
$$

with continuous and dense injection for $1<p<\infty$, see [2, this basis can be extended on $L^{2}(\Omega)$ as a basis of Galerkin to Laplacian operator.

In fact, from Sobolev immersion we have

$$
W_{0}^{\nu, q}(\Omega) \hookrightarrow W_{0}^{\nu-k, q_{k}}(\Omega), \frac{1}{q_{k}}=\frac{1}{q}-\frac{k}{n} .
$$

Choosing $q_{k}=p, \nu-k=1$ and $q=2$ we get

$$
\nu=1+\frac{n}{2}-\frac{n}{p}=1+\frac{n(p-2)}{2 p}>0
$$

and we obtain a Hilbert Space $H_{0}^{\nu}(\Omega)$ such that

$$
H_{0}^{\nu}(\Omega)=W_{0}^{\nu, 2}(\Omega) \hookrightarrow W_{0}^{1, p}(\Omega) .
$$

Let $s$ an integer for which $s>\nu$. We have

$$
H_{0}^{s}(\Omega) \hookrightarrow W_{0}^{1, p}(\Omega) \hookrightarrow H_{0}^{1}(\Omega) \hookrightarrow L^{2}(\Omega) .
$$

By Rellich-Kondrachov theorem, $H_{0}^{1}(\Omega) \hookrightarrow L^{2}(\Omega)$ is compact, so the immersion $H_{0}^{s}(\Omega) \hookrightarrow L^{2}(\Omega)$ is also. From spectral theory, there exists a operator defined by

$$
\left\{H_{0}^{s}(\Omega), L^{2}(\Omega),((\cdot, \cdot))_{H_{0}^{s}(\Omega)}\right\}
$$

and a sequence of eigenvectors $\left(v_{j}\right)_{j \in \mathbb{N}}$ of this operator, such that

$$
\left(\left(v_{j}, v\right)\right)_{H_{0}^{s}(\Omega)}=\lambda_{j}\left(v_{j}, v\right) \text {, for all } v \in H_{0}^{s}(\Omega)
$$

with $\lambda_{j}>0, \lambda_{j} \leq \lambda_{j+1}$, and $\lambda_{j} \rightarrow+\infty$ as $j \rightarrow+\infty$. Moreover $\left(v_{j}\right)_{j \in \mathbb{N}}$ is a complete orthonormal system in $L^{2}(\Omega)$ and $\left(w_{j}=\frac{v_{j}}{\sqrt{\lambda_{j}}}\right)_{j \in \mathbb{N}}$ is a complete orthonormal system in $H_{0}^{s}(\Omega)$. Then $\left(w_{j}\right)_{j \in \mathbb{N}}$ yields a "Galerkin basis" for both $W_{0}^{1, p}(\Omega)$ and $L^{2}(\Omega)$.

\section{Global Solution}

\subsection{Existence.}

Theorem 5.1. Given $u_{0} \in V, u_{1} \in L^{2}(\Omega)$ there exists a function

$$
u: \Omega \times(0, T) \rightarrow \mathbb{R}
$$


such that

$$
\begin{aligned}
& u \in L^{\infty}\left(0, T ; W_{0}^{1, p}(\Omega)\right), \quad u^{\prime} \in L^{\infty}\left(0, T ; L^{2}(\Omega)\right), \\
& u(x, 0)=u_{0}(x), u_{t}(x, 0)=u_{1}(x) \text { a.e. in } \Omega, \\
& \frac{\mathrm{d}}{\mathrm{d} t}\left(u_{t}, v\right)+\left\langle-\Delta_{p} u, v\right\rangle_{p}+(-\Delta u, v)+(g * \Delta u, v)=0, \forall v \in W_{0}^{1, p}(\Omega) \text { in } D^{\prime}(0, T) .
\end{aligned}
$$

Proof. Now, for each $m \in \mathbb{N}$, let us put $V_{m}=\operatorname{Span}\left\{w_{1}, w_{2}, \ldots, w_{m}\right\}$. We search for a function $u_{m}(t)=\sum_{j=1}^{m} k_{j m}(t) w_{j}$ such that for any $v \in V_{m}, u_{m}(t)$ satisfies the approximate equation

$$
\left(u_{m}^{\prime \prime}(t), v\right)+\left\langle-\Delta_{p} u_{m}(t), v\right\rangle_{p}+\left(-\Delta u_{m}(t), v\right)+\left(g * \Delta u_{m}(t), v\right)=0
$$

with the initial conditions $u_{m}(0)=u_{0 m}$ and $u_{m}^{\prime}(0)=u_{1 m}$, where $u_{0 m}$ e $u_{1 m}$ are closed in $V_{m}$ so that

$$
w_{0 m} \rightarrow u_{0} \in W_{0}^{1, p}(\Omega) \text { and } u_{1 m} \rightarrow u_{1} \text { in } L^{2}(\Omega) .
$$

Putting $v=w_{i}, i=1,2, \ldots, m$, and using

$$
\begin{aligned}
u_{m}^{\prime \prime}(t) & =\sum_{j=1}^{m} k_{j m}^{\prime \prime}(t) w_{j}(x), \\
\Delta u_{m}(t) & =\sum_{j=1}^{m} k_{j m}(t) \Delta w_{j}(x), \\
\Delta_{p} u_{m}(t) & =\sum_{j=1}^{m} k_{j m}(t) \Delta_{p} w_{j}(x), \\
\left(g * \Delta u_{m}\right)(t) & =\sum_{j=1}^{m}\left(g * k_{j m}\right)(t) \Delta w_{j}(x),
\end{aligned}
$$

we observe that (8) is a system of ODEs in the variable $t$ and has a local solution $u_{m}(t)$ in a interval $\left[0, t_{m}\right)$, by virtue of Carathéodory's theorem, see [24]. In the next step we obtain priori estimates for the solution $u_{m}(t)$ so that it can be extended to the whole interval $[0, T], T>0$.

Priori Estimates: We replace $v=u_{m}^{\prime}(t)$ in the approximate equation (8) and we get

$$
\left(u_{m}^{\prime}(t), u_{m}^{\prime}(t)\right)-\left\langle\Delta_{p} u_{m}(t), u_{m}^{\prime}(t)\right\rangle_{p}-\left(\Delta u_{m}(t), u_{m}^{\prime}(t)\right)+\left(g * \Delta u_{m}(t), u_{m}^{\prime}(t)\right)=0
$$

Let $\theta \in D\left(0, t_{m}\right)$. We denote by $\langle\cdot, \cdot\rangle$ the duality pairing between $D^{\prime}$ and $D$. So we have,

$$
\begin{gathered}
\left\langle\left(u_{m}^{\prime \prime}(t), u_{m}^{\prime}(t)\right), \theta\right\rangle=\left\langle\frac{1}{2} \frac{\mathrm{d}}{\mathrm{d} t}\left|u_{m}^{\prime}(t)\right|_{2}^{2}, \theta\right\rangle \\
\left\langle\left\langle-\Delta_{p} u_{m}(t), u_{m}^{\prime}(t)\right\rangle_{p}, \theta\right\rangle=\left\langle\frac{1}{p} \frac{\mathrm{d}}{\mathrm{d} t}\left\|\nabla u_{m}(t)\right\|_{p}^{p}, \theta\right\rangle
\end{gathered}
$$




$$
\left\langle\left(-\Delta u_{m}(t), u_{m}^{\prime}(t)\right), \theta\right\rangle=\left\langle\frac{1}{2} \frac{\mathrm{d}}{\mathrm{d} t}\left|\nabla u_{m}(t)\right|_{2}^{2}, \theta\right\rangle
$$

Now, note that

$$
\left(g * \Delta u_{m}(t), u_{m}^{\prime}(t)\right)=-\left(\left(g * \nabla u_{m}\right)(t), \nabla u_{m}^{\prime}(t)\right) .
$$

By Lemma 3.2 we have

$$
\begin{aligned}
2\left(\left(g * \nabla u_{m}\right)(t), \nabla u_{m}^{\prime}(t)\right)= & \int_{\Omega}\left(g^{\prime} \square \nabla u_{m}\right)(t) \mathrm{d} x-g(t) \int_{\Omega}\left|\nabla u_{m}(t)\right|^{2} \mathrm{~d} x \\
& -\int_{\Omega} \frac{\mathrm{d}}{\mathrm{d} t}\left[\left(g \square \nabla u_{m}\right)(t)-\left(\int_{0}^{t} g(s) \mathrm{d} s\right)|\nabla u(t)|^{2}\right] \mathrm{d} x .
\end{aligned}
$$

Then,

$$
\begin{aligned}
\left\langle\left(g * \Delta u_{m}(t), u_{m}^{\prime}(t)\right), \theta\right\rangle=\langle & -\frac{1}{2} \int_{\Omega}\left(g^{\prime} \square \nabla u_{m}\right)(t) \mathrm{d} x+\frac{1}{2} g(t)\left|\nabla u_{m}(t)\right|_{2}^{2} \\
& \left.+\frac{1}{2} \frac{\mathrm{d}}{\mathrm{d} t} \int_{\Omega}\left(g \square \nabla u_{m}\right)(t) \mathrm{d} x-\left(\int_{0}^{t} g(s) \mathrm{d} s\right)\left|\nabla u_{m}(t)\right|_{2}^{2}, \theta\right\rangle .
\end{aligned}
$$

Replacing (10, 11), 12), 13) in (9) we obtain in $D^{\prime}\left(0, t_{m}\right)$

$$
\begin{aligned}
& \frac{\mathrm{d}}{\mathrm{d} t}\left\{\frac{1}{2}\left|u_{m}^{\prime}(t)\right|_{2}^{2}+\frac{1}{p}\left\|\nabla u_{m}(t)\right\|_{p}^{p}+\frac{1}{2}\left(g \square \nabla u_{m}\right)(t)+\frac{1}{2}\left(1-\int_{0}^{t} g(s) \mathrm{d} s\right)\left|\nabla u_{m}(t)\right|_{2}^{2}\right\} \\
= & \frac{1}{2} \int_{\Omega}\left(g^{\prime} \square \nabla u_{m}\right)(t) \mathrm{d} x-\frac{1}{2} g(t)\left|\nabla u_{m}(t)\right|_{2}^{2}
\end{aligned}
$$

The approximate energy

$$
E_{m}(t)=\frac{1}{2}\left|u_{m}^{\prime}(t)\right|_{2}^{2}+\frac{1}{p}\left\|\nabla u_{m}(t)\right\|_{p}^{p}+\frac{1}{2}\left(1-\int_{0}^{t} g(s) \mathrm{d} s\right)\left|\nabla u_{m}(t)\right|_{2}^{2}+\frac{1}{2}\left(g \square \nabla u_{m}\right)(t)
$$

satisfies

$$
\frac{\mathrm{d}}{\mathrm{d} t} E_{m}(t) \leq-\frac{c_{1}}{2} \int_{\Omega}\left(g \square \nabla u_{m}\right)(t) \mathrm{d} x-\frac{1}{2} g(t)\left|\nabla u_{m}(t)\right|_{2}^{2} .
$$

Then $E_{m}(t) \leq E_{m}(0)$. Due to convergence of initial data, there exists a constant $c>0$ independent of $t$ and $m$ such that $E_{m}(t) \leq c$. With this estimate we can extend the aproximate solutions $u_{m}(t)$ to the interval $[0, T]$, see [25], and we have

$$
\begin{gathered}
u_{m}(t) \text { is bounded in } L^{\infty}\left(0, T ; W_{0}^{1, p}(\Omega)\right), \\
u_{m}^{\prime}(t) \text { is bounded in } L^{\infty}\left(0, T ; L^{2}(\Omega)\right), \\
-\Delta_{p} u_{m}(t) \text { is bounded in } L^{\infty}\left(0, T ; W^{-1, p^{\prime}}(\Omega)\right) .
\end{gathered}
$$

From (15) and Lemma 3.1 we deduce

$$
\left(g * \nabla u_{m}\right)(t) \text { is bounded in } L^{\infty}\left(0, T ; L^{2}(\Omega)\right) .
$$

Passage to the Limit: From (15, 16), 18) going to the subsequence if necessary, there exists $u$ such that

$$
u_{m} \rightarrow u \text { weakly star in } L^{\infty}\left(0, T ; W_{0}^{1, p}(\Omega)\right)
$$




$$
\begin{aligned}
u_{m}^{\prime} & \rightarrow u^{\prime} \text { weakly star in } L^{\infty}\left(0, T ; L^{2}(\Omega)\right) \\
g * \nabla u_{m} & \rightarrow g * \nabla u \text { weakly star in } L^{\infty}\left(0, T ; L^{2}(\Omega)\right)
\end{aligned}
$$

and in view of (17) there exists $\mathcal{X}$ such that

$$
-\Delta_{p} u_{m}(t) \rightarrow \mathcal{X} \text { weakly in } L^{\infty}\left(0, T ; W^{-1, p^{\prime}}(\Omega)\right) .
$$

With these convergence we can pass to the limit in the approximate equation (8) see [26, 27], and then

$$
\frac{\mathrm{d}}{\mathrm{d} t}\left(u^{\prime}(t), v\right)+\langle\mathcal{X}(t), v\rangle_{p}+(-\Delta u(t), v)+((g * \nabla u)(t), v)=0,
$$

for all $v \in W_{0}^{1, p}(\Omega)$ in the sense of distributions.

For $x, y \in \mathbb{R}$ and $p \geq 2$, consider the elementary inequalities

$$
\begin{gathered}
\left.|| x\right|^{\frac{p-2}{2}} x-|y|^{\frac{p-2}{2}} y\left|\leq C\left(|x|^{\frac{p-2}{2}}+|y|^{\frac{p-2}{2}}\right)\right| x-y \mid \\
\left.|| x\right|^{p-2} x-|y|^{p-2} y\left|\leq C\left(|x|^{\frac{p-2}{2}}+|y|^{\frac{p-2}{2}}\right)\right||x|^{\frac{p-2}{2}} x-|y|^{\frac{p-2}{2}} y \mid .
\end{gathered}
$$

The inequality $(23)$ is a consequence of the mean value theorem and $(24)$ can be found in 28. As in 29] applying 23], 24) and Hölder generalized inequality with

$$
\frac{p-2}{4 p}+\frac{p-2}{4 p}+\frac{1}{2}+\frac{1}{p}=1
$$

we deduce for all $v \in W_{0}^{1, p}(\Omega)$

$$
\left|\int_{0}^{T}\left\langle-\Delta_{p} u_{m}(t), v\right\rangle_{p}-\left\langle-\Delta_{p} u(t), v\right\rangle_{p} \mathrm{~d} t\right| \leq c \int_{0}^{T}\left|\nabla u_{m}(t)-\nabla u(t)\right|_{2} \mathrm{~d} t .
$$

Now we are going to obtain an estimate for $u_{m}^{\prime \prime}(t)$. Since our Galerkin basis was taken in the Hilbert space $L^{2}(\Omega)$ we can use the standard projection arguments as described in Lions [26]. Then from the approximate equation and the estimates (15)-17) we get

$$
u_{m}^{\prime \prime}(t) \quad \text { is bounded in } \quad L^{\infty}\left(0, T ; W^{-1, q}(\Omega)\right) .
$$

Applying the Lions-Aubin compactness we get from $[19,20$ and 25 ,

$$
\begin{gathered}
u_{m}(t) \rightarrow u(t) \text { strongly in } L^{2}\left(0, T ; L^{2}(\Omega)\right), \\
u_{m}^{\prime}(t) \rightarrow u^{\prime}(t) \text { strongly in } L^{2}\left(0, T ; L^{2}(\Omega)\right) .
\end{gathered}
$$

Using 26) we get that $u_{m}(t) \rightarrow u(t)$ almost everewhere in $\Omega \times(0, T)$ and we have,

$$
-\Delta_{p} u_{m}(t) \rightarrow-\Delta_{p} u(t) \text { weakly in } L^{\infty}\left(0, T ; W^{-1, p^{\prime}}(\Omega)\right) .
$$

From (22), 28) and uniqueness of the limit we conclude that $\mathcal{X}(t)=-\Delta_{p} u(t)$. The verification of the initial data is a routine procedure. The prove of existence is complete. 
5.2. Uniqueness. Let $u$ and $v$ be solutions of (2)-(4) such that

$$
u(x, 0)=u_{0}=v(x, 0) \quad \text { and } \quad u_{t}(x, 0)=u_{1}=v_{t}(x, 0) .
$$

Denoting $w=u-v$ we have

$$
\begin{gathered}
u_{t t}-\Delta w=\Delta_{p} u-\Delta_{p} v-g * \Delta w, \quad \text { in } \Omega \times[0, \infty), \\
w(x, 0)=0, u_{t}(x, 0)=0, x \in \Omega, \\
w(x, t)=0 \quad \text { on } \quad \partial \Omega \times[0, \infty) .
\end{gathered}
$$

We will use the Višik-Ladyenskaya method [30]. Consider for each $\eta \in[0, T]$ the following function

$$
\psi(x, t)=\left\{\begin{array}{cc}
-\int_{t}^{\eta} w(x, \xi) \mathrm{d} \xi & 0 \leq t<\eta, \\
0 & , \quad \eta \leq t \leq T .
\end{array}\right.
$$

Then,

$$
\psi_{t}(x, t)=\left\{\begin{array}{cc}
w(x, t) & , \quad 0 \leq t<\eta, \\
0, & \eta \leq t \leq T .
\end{array}\right.
$$

As $w \in L^{\infty}\left(0, T ; W_{0}^{1, p}(\Omega)\right), w_{t} \in L^{\infty}\left(0, T ; L^{2}(\Omega)\right)$ we have

$$
\psi, \psi_{t} \in L^{\infty}\left(0, T ; L^{2}(\Omega)\right) .
$$

Mutiplying 29 by $\psi$ and performing integration on $\Omega$

$$
\left(w_{t t}, \psi\right)+(\nabla w, \nabla \psi)=\left\langle\Delta_{p} u-\Delta_{p} v, \psi\right\rangle_{p}-(g * \Delta w, \psi) .
$$

Integrating in $[0, \eta]$ and taking into account that $\psi(x, t) \equiv 0$ for all $t \in[\eta, T]$, we have

$\left.\int_{0}^{\eta}\left(w_{t t}, \psi\right) \mathrm{d} t+\int_{0}^{\eta}(\nabla w, \nabla \psi) \mathrm{d} t=\int_{0}^{\eta}\left\langle\Delta_{p} u-\Delta_{p} v, \psi\right\rangle_{p} \mathrm{~d} t-\int_{0}^{\eta}(g * \Delta w), \psi\right) \mathrm{d} t$.

As $\psi(\eta)=w(0)=0$ we get

$-\int_{0}^{\eta}\left(w_{t}, \psi_{t}\right) \mathrm{d} t+\int_{0}^{\eta}(\nabla w, \nabla \psi) \mathrm{d} t=\int_{0}^{\eta}\left\langle\Delta_{p} u-\Delta_{p} v, \psi\right\rangle_{p} \mathrm{~d} t-\int_{0}^{\eta}((g * \Delta w)(t), \psi) \mathrm{d} t$.

From (31), 32, and (33)

$-\int_{0}^{\eta}\left(w_{t}, w\right) \mathrm{d} t+\int_{0}^{\eta}\left(\nabla \psi_{t}, \nabla \psi\right) \mathrm{d} t=\int_{0}^{\eta}\left\langle\Delta_{p} u-\Delta_{p} v, \psi\right\rangle_{p} \mathrm{~d} t-\int_{0}^{\eta}(g * \Delta w, \psi) \mathrm{d} t$.

That is

$-\frac{1}{2} \int_{0}^{\eta} \frac{\mathrm{d}}{\mathrm{d} t}|w|_{2}^{2} \mathrm{~d} t+\frac{1}{2} \int_{0}^{\eta} \frac{\mathrm{d}}{\mathrm{d} t}|\nabla \psi|_{2}^{2} \mathrm{~d} t=\int_{0}^{\eta}\left\langle\Delta_{p} u-\Delta_{p} v, \psi\right\rangle_{p} \mathrm{~d} t-\int_{0}^{\eta}((g * \Delta w)(t), \psi) \mathrm{d} t$,

that implies

$$
-\frac{1}{2}|w(\eta)|_{2}^{2}-\frac{1}{2}|\nabla \psi(0)|_{2}^{2} \leq \int_{0}^{\eta}\left\langle\Delta_{p} u-\Delta_{p} v, \psi\right\rangle_{p} \mathrm{~d} t-\int_{0}^{\eta}((g * \Delta w)(t), \psi) \mathrm{d} t
$$


As before, applying (23), 24) and Hölder generalized inequality with

$$
\frac{p-2}{4 p}+\frac{p-2}{4 p}+\frac{1}{p}+\frac{1}{2}=1
$$

we obtain

$$
\left|\left\langle\Delta_{p} u-\Delta_{p} v, \psi\right\rangle_{p}\right| \leq c|\nabla \psi|_{2} .
$$

From (34) and continuous and dense injection (7) we deduce that $g * \Delta w \in$ $L^{2}\left(0, T ; L^{2}(\Omega)\right)$. Then

$$
\left|\int_{\Omega}(g * \Delta w)(t) \psi \mathrm{d} x\right| \leq\left(\int_{\Omega}|g * \Delta w|^{2} \mathrm{~d} x\right)^{\frac{1}{2}}\left(\int_{\Omega}|\psi|^{2} \mathrm{~d} x\right)^{\frac{1}{2}} \leq c|\psi|_{2} .
$$

From (35), 36), 37), Poincarè and Cauchy-Schwarz inequalities we deduce

$$
\frac{1}{2}|w|_{2}^{2}+\frac{1}{2}|\nabla \psi(0)|_{2}^{2} \leq c \int_{0}^{\eta}|\nabla \psi|_{2}^{2} \mathrm{~d} t
$$

Now we introduce $w_{1}(x, t)=\int_{0}^{t} w(x, \xi) \mathrm{d} \xi$, for all $t \in[0, \xi)$ we have

$\psi(x, t)=-\int_{0}^{\eta} w(x, \xi) \mathrm{d} \xi=-\int_{0}^{\eta} w(x, \xi) \mathrm{d} \xi+\int_{0}^{t} w(x, \xi) \mathrm{d} \xi=w_{1}(x, t)-w_{1}(x, \eta)$,

and then

$$
\psi(x, 0)=w_{1}(x, 0)-w_{1}(x, \eta)=-w_{1}(x, \eta) .
$$

From (38), 39 and 40 we obtain

$$
\frac{1}{2}\left|\nabla w_{1}\right|_{2}^{2} \leq c \int_{0}^{\eta}\left|\nabla w_{1}\right|_{2}^{2} \mathrm{~d} t .
$$

By Gronwall's inequality we conclude $\left|\nabla w_{1}\right|_{2}^{2} \leq 0$. By $(39)$ we deduce that $\nabla \psi=0$ in $L^{2}(\Omega)$ for all $t \in[0, T]$. Finally follows from $\left[38\right.$ that $|w|_{2}^{2} \leq 0$ and then $u=v$ in $L^{2}(\Omega)$ for all $t \in[0, T]$.

\section{Asymptotic Behaviour}

Theorem 6.1. Let $u$ be a solution of (2)-(4) with initial data $u_{0} \in V, u_{1} \in$ $L^{2}(\Omega)$. For $\phi: \mathbb{R}_{+} \rightarrow \mathbb{R}_{+}$a increasing $C^{2}$ function such that $\phi(0)=0$ and $\phi(t) \stackrel{t \rightarrow+\infty}{\longrightarrow}+\infty$ we have for $q>0$

$$
E(t) \leq c E(0)(1+\phi(t))^{\frac{-1}{q}}, \forall t>0
$$

where $c$ is a positive constant independent of the initial energy $E(0)$.

Proof. We will use Lemma 3.3 due to P. Martinez 16 based on a new inequality that generalizes a result of Haraux [17. For the goal we start the proof of (41) multiplying 2 by $E^{q}(t) \phi^{\prime}(t) u$ and so we set

$$
\int_{0}^{T} E^{q} \phi^{\prime} \int_{\Omega} u\left(u_{t t}-\Delta_{p} u-\Delta u+g * \Delta u\right) \mathrm{d} x \mathrm{~d} t=0,
$$


from where we obtain by straight calculations

$$
\begin{aligned}
2 \int_{S}^{T} E^{q+1} \phi^{\prime} \mathrm{d} t \leq & -\left[E^{q} \phi^{\prime} \int_{\Omega} u u_{t} \mathrm{~d} x\right]_{S}^{T} \\
& +4 \int_{S}^{T}\left[\left(q E^{\prime} E^{q-1} \phi^{\prime}+E^{q} \phi^{\prime \prime}\right) \int_{\Omega} u u_{t} \mathrm{~d} x\right] \mathrm{d} t \\
& +4 \int_{S}^{T}\left[E^{q} \phi^{\prime} \frac{1}{2} \int_{\Omega}\left|u_{t}\right|^{2} \mathrm{~d} x\right] \mathrm{d} t+\int_{S}^{T}\left[E^{q} \phi^{\prime} \frac{1}{2} \int_{\Omega} g \square \nabla u \mathrm{~d} x\right] \mathrm{d} t \\
& +\int_{S}^{T}\left[E^{q} \phi^{\prime} \frac{1}{2} \int_{\Omega} \int_{0}^{t} g(t-s)|\nabla u(s)|^{2} \mathrm{~d} s \mathrm{~d} x\right] \mathrm{d} t \\
& +\int_{S}^{T}\left[E^{q} \phi \frac{1}{2} \int_{\Omega}|\nabla u|^{2} \mathrm{~d} x \int_{0}^{T} g(t-s) \mathrm{d} s\right] \mathrm{d} t
\end{aligned}
$$

In the stability set $V$ we have

$$
\frac{1}{2} \int_{0}^{t} g(t-s)|\nabla u(s)|^{2} \mathrm{~d} s \leq \frac{1}{p}\|\nabla u(s)\|_{p}^{p} .
$$

Replacing 43 in 42 we obtain

$$
\begin{aligned}
\int_{S}^{T} E^{q+1} \phi^{\prime} \mathrm{d} t \leq & -\left[E^{q} \phi^{\prime} \int_{\Omega} u u_{t} \mathrm{~d} x\right]_{S}^{T}+\int_{0}^{T}\left[\left(q E^{\prime} E^{q-1} \phi^{\prime}+E^{q} \phi^{\prime \prime}\right) \int_{\Omega} u u_{t} \mathrm{~d} x\right] \mathrm{d} t \\
& +\frac{3}{2} \int_{S}^{T} E^{q} \phi^{\prime} \int_{\Omega}\left|u_{t}\right|^{2} \mathrm{~d} x \mathrm{~d} t
\end{aligned}
$$

Now, we will estimate each term on the right side of 444). Applying the same argument as in [6] we deduce

$$
\left|\left[E^{q} \phi^{\prime} \int_{\Omega} u u_{t} \mathrm{~d} x\right]_{S}^{T}\right| \leq c E(s), \forall t \geq S
$$

and

$$
\left|\int_{S}^{T}\left[\left(q E^{\prime} E^{q-1} \phi^{\prime}+E^{q} \phi^{\prime \prime}\right) \int_{\Omega} u u_{t} \mathrm{~d} x\right] \mathrm{d} t\right| \leq c E(s), \forall t \geq S .
$$

The estimate of $\int_{S}^{T} E^{q} \phi^{\prime} \int_{\Omega}\left|u_{t}\right|^{2} \mathrm{~d} x \mathrm{~d} t$ is quite delicate. Let $\sigma: \mathbb{R}_{+} \rightarrow \mathbb{R}_{+}$be a strictly positive function such that $\int_{0}^{\infty} \sigma(t) \mathrm{d} \tau=+\infty . \quad \phi(t)=\int_{0}^{t} \sigma(\tau) \mathrm{d} \tau$ satisfies $\phi(0)=0$ and $\phi(t) \stackrel{t \rightarrow+\infty}{\longrightarrow}+\infty$. Consider $\rho(t, u) \leq-E^{\prime}(t)$. 
According to the [6] for all $0<S<T$ ande $l<m+1$

$$
\begin{aligned}
\int_{S}^{T} E^{q} \phi^{\prime} \int_{\Omega}\left|u_{t}\right|^{l} \mathrm{~d} x \mathrm{~d} t \leq & c \int_{S}^{T} E^{q} \phi^{\prime} \int_{\Omega} \frac{1}{\sigma(t)} u_{t} \rho(t, u) \mathrm{d} x \mathrm{~d} t \\
& +c^{\prime} \int_{S}^{T} E^{q} \phi^{\prime} \int_{\Omega}\left(\frac{1}{\sigma(t)} u_{t} \rho(t, u)\right)^{\frac{l}{m+1}} \mathrm{~d} x \mathrm{~d} t \\
\leq & c \int_{S}^{T} E^{q} \frac{\phi^{\prime}}{\sigma(t)}\left(-E^{\prime}\right) \int_{\Omega}\left|u^{\prime}\right| \mathrm{d} x \mathrm{~d} t \\
& +c^{\prime} \int_{S}^{T} E^{q} \phi^{\prime} \sigma^{-\frac{l}{m+1}}(t)\left(-E^{\prime}\right)^{\frac{l}{m+1}} \int_{\Omega}\left|u^{\prime}\right|^{\frac{l}{m+1}} \mathrm{~d} x \mathrm{~d} t .
\end{aligned}
$$

Applying Hölder inequality, using $l<m+1,\left|u^{\prime}\right|_{2}^{2}<c$ we get

$$
\begin{aligned}
\int_{S}^{T} E^{q} \phi^{\prime} \int_{\Omega}\left|u_{t}\right|^{l} \mathrm{~d} x \mathrm{~d} t \leq & c \int_{S}^{T} E^{q} \frac{\phi^{\prime}}{\sigma(t)}\left(-E^{\prime}\right) \mathrm{d} t \\
& +c^{\prime} \int_{S}^{T} E^{q} \phi^{\prime \frac{m+1-l}{m+1}}\left(\frac{\phi^{\prime}}{\sigma(t)}\right)^{\frac{l}{m+1}}\left(-E^{\prime}\right)^{\frac{l}{m+1}} \mathrm{~d} t .
\end{aligned}
$$

For fix and arbitrarily small $\varepsilon>0$ (to be chosen later). By applying Young's inequality $\frac{1}{\frac{m+1}{m+1-l}}+\frac{1}{\frac{m+1}{l}}=1$ we obtain

$$
\begin{aligned}
\int_{S}^{T} E^{q} \phi^{\prime} \int_{\Omega}\left|u^{\prime}\right|^{l} \mathrm{~d} x \mathrm{~d} t \leq & c \int_{S}^{T} E^{q} \frac{\phi^{\prime}}{\sigma(t)}\left(-E^{\prime}\right) \mathrm{d} t \\
& +c^{\prime} \frac{m+1-l}{m+1} \varepsilon^{\frac{m+1}{m+1-l}} \int_{S}^{T} E^{q \frac{m+1}{m+1-l}} \phi^{\prime} \mathrm{d} t \\
& +c^{\prime} \frac{l}{m+1} \int_{\Omega}\left(-E^{\prime}\right) \frac{\phi^{\prime}}{\sigma(t)} \varepsilon^{-\frac{m+1}{l}} \mathrm{~d} t
\end{aligned}
$$

From where follows

$$
\begin{aligned}
\int_{S}^{T} E^{q} \phi^{\prime} \int_{\Omega}\left|u_{t}\right|^{l} \mathrm{~d} x \mathrm{~d} t \leq & c E^{q}(s)+c^{\prime} \frac{m+1-l}{m+1} \varepsilon^{\frac{m+1}{m+1-l}} \int_{S}^{T} E^{q \frac{m+1}{m+1-l}} \phi^{\prime} \mathrm{d} t \\
& +c^{\prime} \frac{l}{m+1} \varepsilon^{-\frac{m+1}{l}} E(s) .
\end{aligned}
$$

Making $l=2, \rho(t, u)=\frac{1}{2} \int_{\Omega}(g \square \nabla u)(t) \mathrm{d} x$ e choosing $q$ such that

we obtain

$$
\frac{m+1}{m+1-l}=\frac{q+1}{q}
$$

$$
\int_{S}^{T} E^{q} \phi^{\prime} \int_{\Omega}\left|u_{t}\right|^{2} \mathrm{~d} x \mathrm{~d} t \leq c E(s)+c \frac{m-2}{m+1} \varepsilon^{\frac{m+1}{m-1}} \int_{S}^{T} E^{q+1} \phi^{\prime} \mathrm{d} t+\frac{2 c}{m+1} \varepsilon^{-\frac{m+1}{2}} E(s)
$$

Now we deduce from (44), 45, ,46) and 47.

$$
\left(1-c \frac{m-1}{m+1} \varepsilon^{\frac{m+1}{m-1}}\right) \int_{S}^{T} E^{q+1} \phi^{\prime} \mathrm{d} t \leq c(\varepsilon) E(s) .
$$


Finally, choosing $\varepsilon$ small enough we concludes

$$
\int_{S}^{T} E^{q+1} \phi^{\prime} \mathrm{d} t \leq c E(s), q>0
$$

and the proof is complete.

\section{Concluding remarks}

When $p=2$ is well known that the equation (2) describes a homogeneous and isotropic viscoelastic solid and the genuine memory $g * \Delta u$ induces a damping mechanism so the asymptotic stability is to be expected. For instance, for the nonhomogeneous problem with function $f$ independent of time and source term the existence of a global attractor was proved in [31. The problem with supercritical source and damping terms was studied in [32. Employing the theory of monotone operators and nonlinear semigroups, combined with energy methods was established the existence of a unique local weak solution in the finite energy space. As follow-up work, recently in 33 was considered supercritical nonlinearities and was studied blow-up of solutions when the source is stronger than dissipation. For The case $p>2$ the nonlinear equation (2) leads to a problem not previously considered. The highlight here was to prove the existence of solution and energy decay in the appropriate set of stability created from the Nehari manifold.

\section{Acknowledgement}

This project was been supported by PNPD/UFBA/CAPES (Brazil). The authors is grateful to the referees for his valuable comments and suggestions that helped improving the original manuscript.

\section{Competing Interests}

The authors declare that they have no competing interests.

\section{REFERENCES}

1. Adams, R. A.(1975). Sobolev Spaces, Academic Press.

2. Brezis, H. (2010). Functional analysis, Sobolev spaces and partial differential equations. Springer Science \& Business Media.

3. M. Dreher, The wave equation for the $p$-Laplacian, Hokkaido Mathematical Journal 36 (2007) 21-52 .

4. Greenberg, J. M., CAMY, R. C. M., \& Mizel, V. J. (1968). On the Existence, Uniqueness, and Stability of Solutions of the Equation $\sigma^{\prime}\left(u_{x}\right) u_{x x}+\lambda u_{x t x}=\rho_{0} u_{t t}$. Journal of Mathematics and Mechanics, 707-728.

5. Ma, T. F., \& Soriano, J. A. (1999). On weak solutions for an evolution equation with exponential nonlinearities. Nonlinear Analysis: Theory, Methods $\&$ Applications, 37(8), 1029-1038.

6. Benaissa, A., \& Mokeddem, S. (2007). Decay estimates for the wave equation of $p$-Laplacian type with dissipation of $m$-Laplacian type. Mathematical methods in the applied sciences, $30(2), 237-247$. 
7. Rammaha, M., Toundykov, D., \& Wilstein, Z. (2012). Global existence and decay of energy for a nonlinear wave equation with $p$-Laplacian damping. Discrete Contin. Dyn. Syst., 32(12), 4361-4390.

8. Pei, P., Rammaha, M. A., \& Toundykov, D. (2015). Weak solutions and blow-up for wave equations of $p$-Laplacian type with supercritical sources. Journal of Mathematical Physics, $56(8), 081503$.

9. Ye, Y. (2007). Global existence and asymptotic behavior of solutions for a class of nonlinear degenerate wave equations. International Journal of Differential Equations, 2007, 19685.

10. Biazutti, A. C. (1995). On a nonlinear evolution equation and its applications. Nonlinear analysis: Theory, Methods \& Applications, 24(8), 1221-1234.

11. Ang, D. D., \& Pham Ngoc Dinh, A. (1988). Strong solutions of a quasilinear wave equation with nonlinear damping. SIAM Journal on Mathematical Analysis, 19(2), 337-347.

12. d'Ancona, P., \& Spagnolo, S. (1991). On the life span of the analytic solutions to quasilinear weakly hyperbolic equations. Indiana University Mathematics Journal,40, 71-99.

13. Chueshov, I., \& Lasiecka, I. (2006). Existence, uniqueness of weak solutions and global attractors for a class of nonlinear $2 D$ Kirchhoff-Boussinesq models. Discrete and Continuous Dynamical Systems, 15(3), 777-809.

14. Zhijian, Y. (2003). Global existence, asymptotic behavior and blowup of solutions for a class of nonlinear wave equations with dissipative term. Journal of Differential Equations, 187(2), 520-540.

15. Gao, H., \& Ma, T. F. (1999). Global solutions for a nonlinear wave equation with the p-Laplacian operator. Electronic Journal of Qualitative Theory of Differential Equations, 1999(11), 1-13.

16. Martinez, P. (1999). A new method to obtain decay rate estimates for dissipative systems. ESAIM: Control, Optimisation and Calculus of Variations, 4, 419-444.

17. Haraux, A. (1985). Two remarks on dissipative hyperbolic problems. Research Notes in Mathematics, 122, 161-179.

18. Nakao, M. (1978). A difference inequality and its application to nonlinear evolution equations. Journal of the Mathematical Society of Japan, 30(4), 747-762.

19. Ambrosetti, A., \& Rabinowitz, P. H. (1973). Dual variational methods in critical point theory and applications. Journal of functional Analysis, 14(4), 349-381.

20. Willem, M. (1997). Minimax theorems(Progress in Nonlinear Differential Equations and Their Applications) (Vol. 24). Springer Science \& Business Media.

21. Alves, M. S., Raposo, C. A., Rivera, J. E. M., Villagrán, M., \& Villagrn, O. V. (2010). Uniform stabilization for the transmission problem of the Timoshenko system with memory. Journal of Mathematical Analysis and Applications, 369(1), 323-345.

22. Coffman, C. V. (1973). Lyusternik-Schnirelman theory and eigenvalue problems for monotone potential operators. Journal of Functional Analysis, 14(3), 237-252.

23. Zeidler, E. Nonlinear Functional Analysis and its Applications II/B, Nonlinear Monotone Operators, 1990. Dumitru Motreanu Département de Mathématiques, Université de Perpignan, 66025 .

24. Coddington, E. A., \& Levinson, N. (1955). Theory of ordinary differential equations. Tata McGraw-Hill Education.

25. Hale, J. K. (1997). Ordinary Differential Equations. Dover Publications, INC.

26. Lions, J. L. (1988). Contrôlabilité exacte, perturbations et stabilisation de systèmes distribués. Tome 1. RMA, 8. .

27. Zhijian, Y. (2009). Longtime behavior for a nonlinear wave equation arising in elasto-plastic flow. Mathematical Methods in the Applied Sciences, 32(9), 1082-1104.

28. Domokos, A., \& Manfredi, J. J. (2009). A second order differentiability technique of Bojarski-Iwaniec in the Heisenberg group. Functiones et Approximatio Commentarii Mathematici, 40(1), 69-74. 
29. Raposo, C. A., Ribeiro, J. O., \& Cattai, A. P.(2018). Global solution for a thermoelastic system with -Laplacian. Applied Mathematics Letters, 86, 119-125.

30. Vishik, M. I., \& Ladyzhenskaya, O. A. (1956). Boundary value problems for partial differential equations and certain classes of operator equations. Uspekhi matematicheskikh nauk, 11(6), 41-97.

31. Giorgi, C., Rivera, J. E. M., \& Pata, V. (2001). Global attractors for a semilinear hyperbolic equation in viscoelasticity. Journal of Mathematical Analysis and Applications, 260(1), 83-99.

32. Guo, Y., Rammaha, M. A., Sakuntasathien, S., Titi, E. S., \& Toundykov, D. (2014). Hadamard well-posedness for a hyperbolic equation of viscoelasticity with supercritical sources and damping. Journal of Differential Equations, 257(10), 3778-3812.

33. Guo, Y., Rammaha, M. A., \& Sakuntasathien, S. (2017). Blow-up of a hyperbolic equation of viscoelasticity with supercritical nonlinearities. Journal of Differential Equations, 262(3), 1956-1979.

\section{Carlos Alberto Raposo}

Mathematics Department, Federal University of São João del-Rey 36307-352 São João del-Rey-MG, Brasil.

e-mail: raposo@ufsj.edu.br

\section{Adriano Pedreira Cattai}

Mathematics Department, State University of Bahia 41150-000 Salvador-BA, Brasil.

e-mail: cattai@uneb.br

\section{Joilson Oliveira Ribeiro}

Mathematics Department, Federal University of Bahia 40170-110 Salvador-BA, Brasil.

e-mail: joilsonor@ufba.br 